
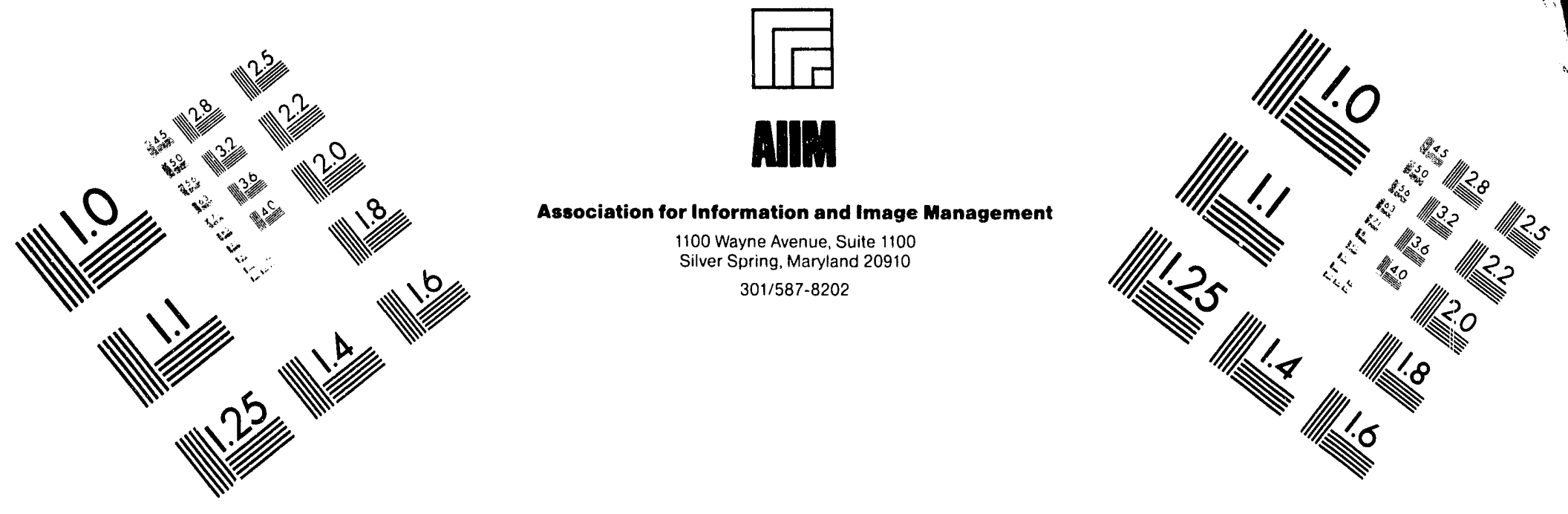

Centimeter

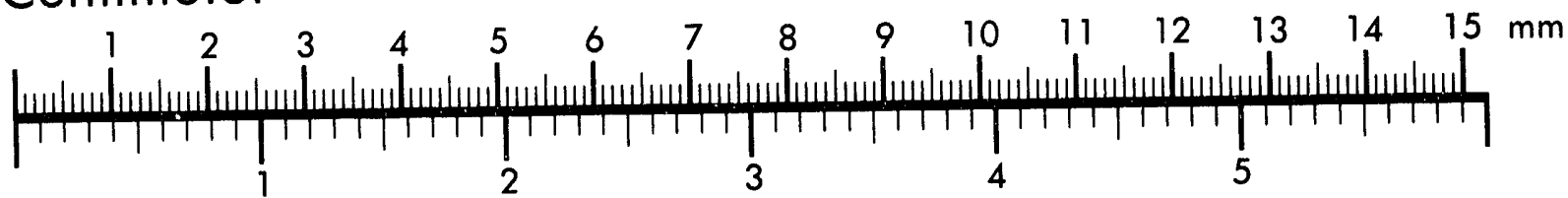
Inches
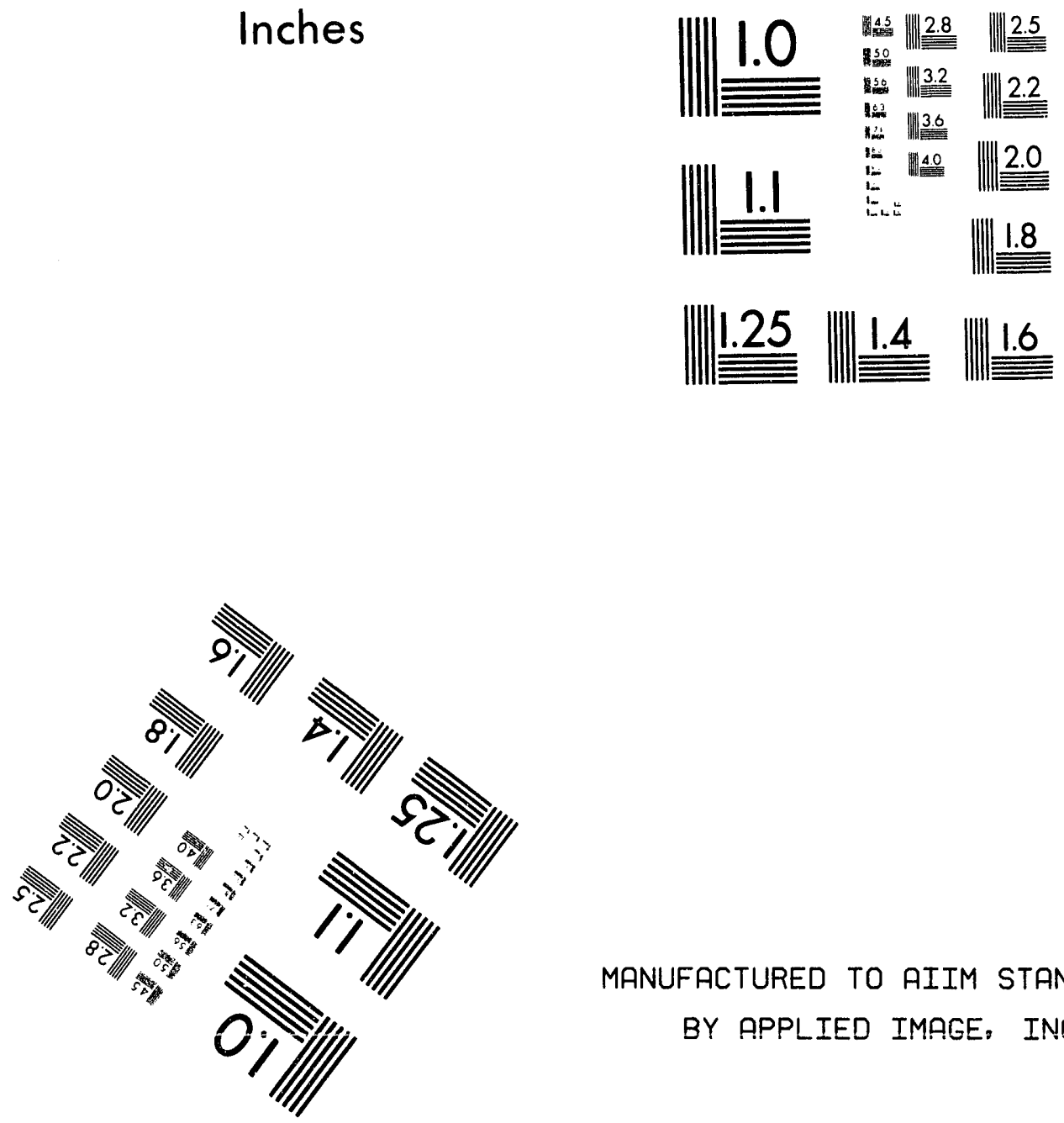

MANUFACTURED TO AIIM STANDARDS

BY APPLIED IMAGE, INC.

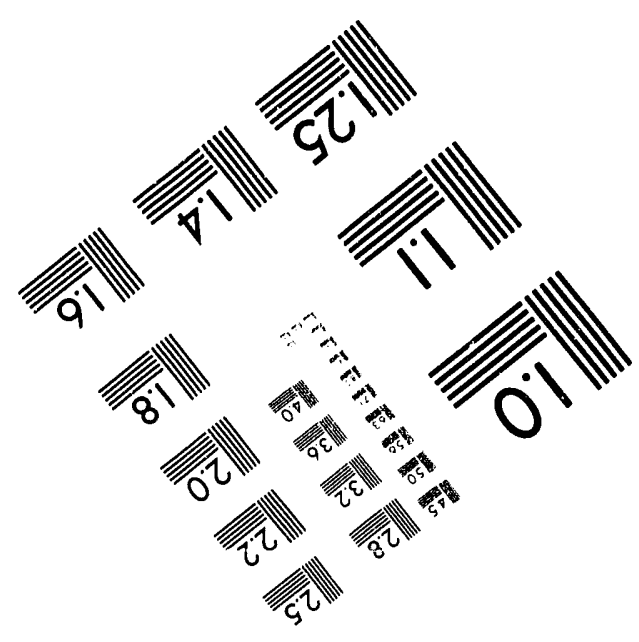



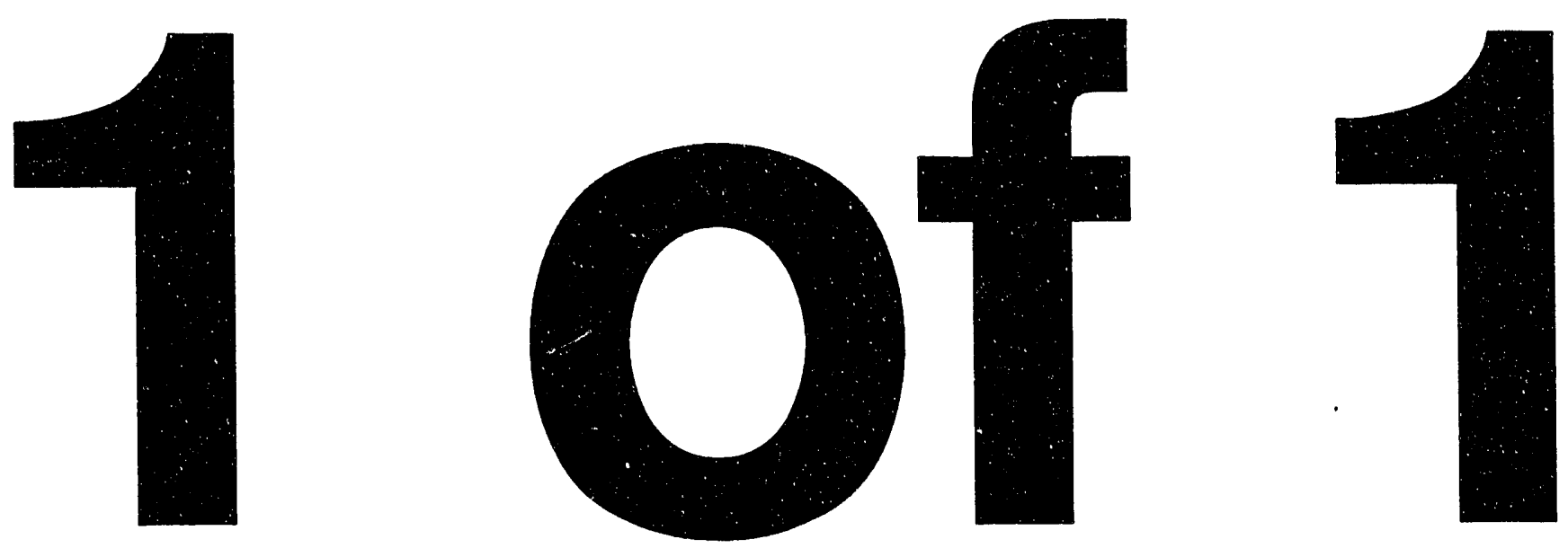


\title{
An Optimized Formulation for Deprit-Type Lie Transformations of Taylor Maps for Symplectic Systems
}

\author{
Jicong Shi ${ }^{1}$ \\ Department of Physics, University of Houston, Houston, TX 77204-5506, USA \\ Yiton T' Yan \\ SSC Laboratory ${ }^{2}, 2550$ Beckleymeade Ave., Dallas, TX 75237, USA
}

\begin{abstract}
We present an optimized iterative formulation for directly transforming a Taylor map of a symplectic system into a Deprit-type Lie transformation, which is a composition of a linear transfer matrix and a single Lie transformation, to an arbitrary order.
\end{abstract}

For a sympletic system, a one-turn map can be written as a composition of a linear transfer matrix and a nonlinear Taylor map $M$ of the form [1]

$$
M \vec{z}=\vec{z}+\vec{U}_{2}(\vec{z})+\vec{U}_{3}(\vec{z})+\ldots
$$

which can be converted order-by-order into Lie transformations in the form of Dragt-Finn factorization [2]:

$$
M \vec{z}=:^{: f_{3}(\vec{z}):} e^{: f_{4}(\vec{z}): \ldots \vec{z},}
$$

where $\vec{z}$ represents the canonical phase-space coordinates; $f_{i}(\vec{z})$ and $\vec{U}_{i}$ are the homogeneous polynomial and the vectorial homogeneous polynomial of degree $i$, respectively; : $f_{i}(\vec{z})$ : is the Lie operator associated with the function $f_{i}(\vec{z})$, which is defined by the Poisson bracket operation $: f_{i}(\vec{z}): \vec{z}=\left[f_{i}(\vec{z}), \vec{z}\right]$. By means of the Campbell-BakerHausdorff (CBH) formula [2], the product of Lie transformations in Eq. (2) can be combined to form a single Lie transformation:

$$
M \vec{z}=e^{: g(\vec{z}): \vec{z}},
$$

where

$$
g(\vec{z})=g_{3}(\vec{z})+g_{4}(\vec{z})+\ldots,
$$

and $g_{i}(\vec{z})$ is a homogeneous polynomial of order $i$. Note that except $g_{3}(\vec{z})=f_{3}(\vec{z}), g_{i}(\vec{z})$ is generally different from $f_{i}(\vec{z})$. Since obtaining a single Lie transformation from Eq. (2) via $\mathrm{CBH}$ formula is pretty tedious and one may need such a single Lie transformation under certain circumstances [3], we have worked out an optimized algorithmic formulation for obtaining this single Lie transformation directly from the Taylor map of Eq. (1) [4]. It should

\footnotetext{
'Supported by TNRLC under award FCFY9221 and the U.S. Department of Energy under grant DE-FG05-87ER40374.

${ }^{2}$ Operated by the Universities Research Association, Inc., for the U.S. Department of Energy under Contract No. DE-AC35. 89ER4U486.
}

be noted that we are not claiming that we are the first to try such a direct single Lie transformation. It is very likely that others may have different approcich. The purpose of this note is to share with colleagues the simple and optimized algorithm we have obtained. The algorithm is described as follows.

Let us define, for each order $n$, a set of auxiliary vector homogeneous polynomials of order $\mathrm{n},\left\{\vec{W}_{n}^{(m)}(\vec{z}), m=\right.$ $1,2, \ldots, n\} . \quad g_{n+1}(\vec{z})$ for $n=2,3, \ldots$ are then obtained through order-by-order iteration given by the following steps:

$$
g_{n+1}(\vec{z})=-\frac{1}{n+1} \vec{z}^{T} S \vec{W}_{n}^{(1)}(\vec{z})
$$

where

$$
\vec{W}_{2}^{(1)}(\vec{z})=\vec{U}_{2}(\vec{z})
$$

and for $n \geq 3$,

$$
\vec{W}_{n}^{(m)}(\vec{z})=\frac{1}{m} \sum_{i=1}^{n-m}: g_{i+2}(\vec{z}): W_{n-i}^{(m-1)}(\vec{z}),
$$

where $2 \leq m \leq n$ and

$$
\vec{W}_{n}^{(1)}(\vec{z})=\vec{U}_{n}(\vec{z})-\sum_{m=2}^{n-1} \vec{W}_{n}^{(m)}(\vec{z}) .
$$

In Eq. (5), $S$ is the antisymmetric matrix [1] and the superscript $T$ denotes the transpose.

This optimized algorithm is planned to be implemented in Zlib [5], a differential Lie algebraic numerical library.

We would like to thank S.K. Kauffmann for useful discussions and S. Ohnuma for numerous encouragement.

\section{References}

[1] Y.T. Yan, The Physics of Particle Accelerators, AIP Conf. Proc. No. 249, p. 378, M. Month and M. Dienes eds. (1992).

[2] A. Dragt and J. Finn, J. Math. Phys. 17, 2215 (1976).

[3] J. Shi and Y. T. Yan, "Symmetric IntegrablePolynomial Factorization for Symplectic OneTurn-Map Tracking" in these proceedings.

[4] J. Shi and Y. T. Yan, preprint (1993).

[5 ] Y.T. Yan and C. Yan, SSCL-300 (1990). 


\section{DISCLAIMER}

This report was prepared as an account of work sponsored by an agency of the United States Government. Neither the United States Government nor any agency thereof, nor any of their employees, makes any warranty, express or implied, or assumes any legal liability or responsibility for the accuracy, completeness, or usefulness of any information, apparatus, product, or process disclosed, or represents that its use would not infringe privately owned rights. Reference herein to any specific commercial product, process, or service by trade name, trademark, manufacturer, or otherwise does not necessarily constitute or imply its endorsement, recommendation, or favoring by the United States Government or any agency thereof. The views and opinions of authors expressed herein do not necessarily state or reflect those of the United States Government or any agency thereof. 

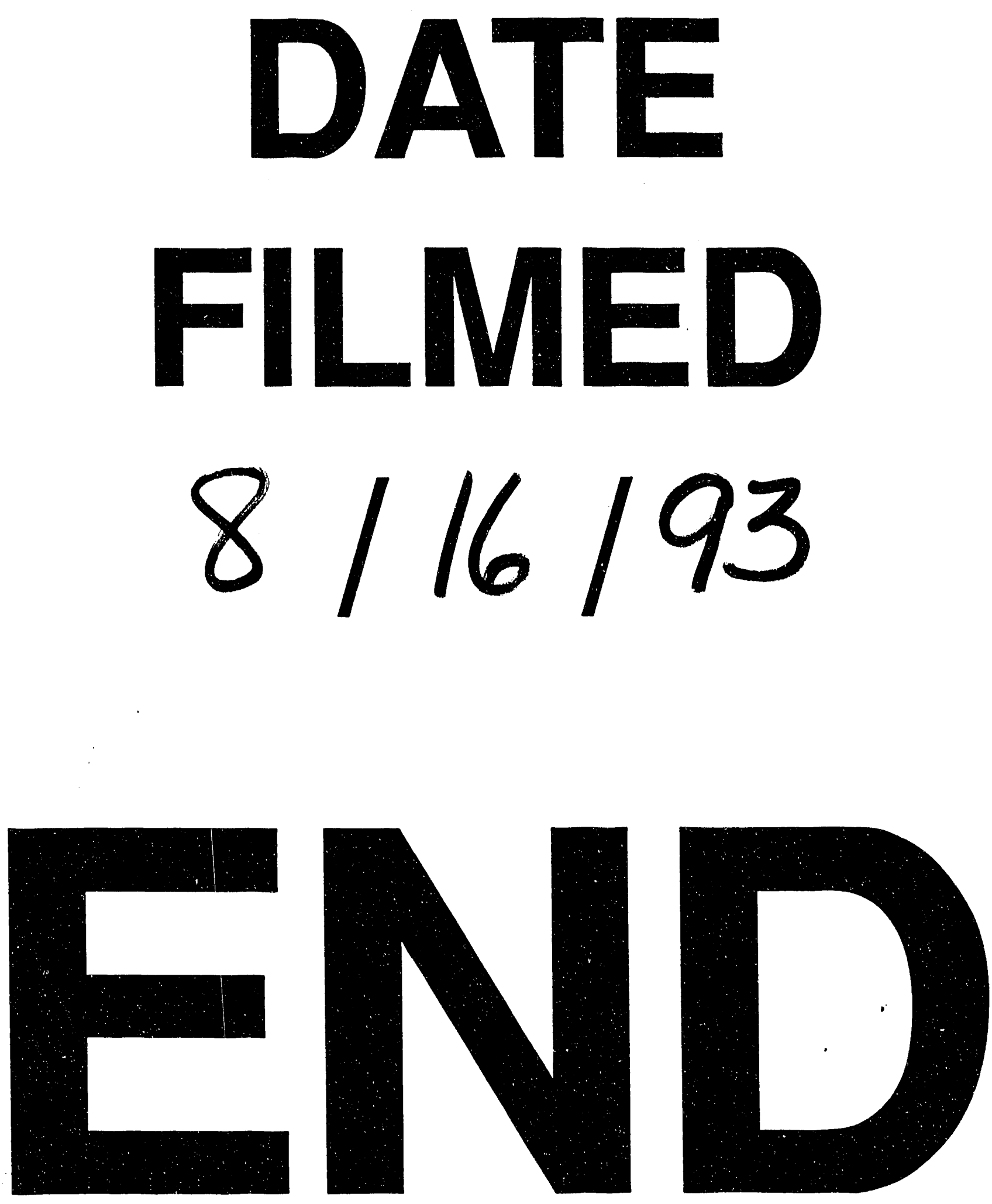
